\title{
A new method to recover the correct land use and public transport interaction
}

\author{
S. Gori, M. Nigro \& M. Petrelli \\ Department of Engineering, Roma Tre University, Italy
}

\begin{abstract}
In the last years the increasing use of private vehicles in urban areas creates negative impacts on the society particularly for the congestion, implying an increase of travel times, of air and noise pollution, of accidents and the excessive production of greenhouse gases and land consumption.

Public transport could represent a more efficient mode of travel respect to car, playing an important role to provide a more sustainable transport system.

However, infrastructural actions operated on the public transport system are usually of long term, respect to actions operated on land use, thus creating a considerable temporal gap between the land use and the transport system development.

Starting from these remarks, the present study proposes a new method to overcome this temporal discrepancy, using the residual capacity of the mass transit system (existing or its short term development) as a variable to indicate the location and the magnitude of new residential and activities developments. The method has been applied to the city of Rome (Italy), suggesting how the Local Authority could guide the development of the urban area in a sustainable way for the next years.
\end{abstract}

Keywords: land use, sustainability, public transport, transit demand estimation.

\section{Introduction}

The increasing use of the car for travel, both in developing and developed countries and the continuous spread of residences and activities in the urban areas, have increased the length of trips and the use of motorized private transport, reducing in many cases the possibilities to develop an efficient public transport system. The impacts are congestion, increase of travel times, of air and 
noise pollution, of the number of accidents, the excessive production of greenhouse gases and the land consumption.

Sustainability and transportation has been heavily dealt with by the research community in the last twenty years centering the attention on the analysis of the interaction between land use and the transport system, the role of the land use characteristics in the travel patterns choice and the importance of the public transport to develop a sustainable transport system.

About the role of public transport, Vuchic [1] states the importance of planning the evolution of the cities taking into account as driving force the relationship between land use and transportation. Banister [2] underlines the importance of the implementation of appropriate policies in order to develop high-quality liveable cities, also encouraging the modal shift to green modes and public transport.

About the opportunities provided by the public transport systems to develop a sustainable mobility, Bernick and Cervero [3] and Cervero [4] show, introducing the concept of the "transit metropolis", examples of transit services that provide respectable alternatives to travel by car; all the examples are characterized by a strong interaction between the land use policy and the transport system planning.

Extensive debates are related to the role played by the population and activities densities to explain the level of car and public transport use: Gori et al. [5] highlight the importance of density of residences and activities, the need for a good quality access system to the transit services stops and the importance of the configuration of the transit network; however, single actions on these variables demonstrate to be not successful due to the complexity of the analysed system.

The present study proposes a new method to effectively exploit the transit network, modifying the usual planning approach: the method would like to overcome the temporal discrepancy between infrastructural actions on land use and infrastructural actions on the transport system, using the residual capacity of the mass transit system (existing or its short term development) as a variable to indicate the location and the magnitude of new residential and activities developments.

The method has been applied to the city of Rome (Italy), suggesting how the Local Authority could guide the development of the urban area in a sustainable way for the next years.

\section{The methodology}

Infrastructural actions on land use, such as the building of new neighborhoods, are usually of short term, while infrastructural actions on the mass transit systems are usually of long term; it happens mainly because the first ones are characterized by private funding due to secure and large profits, while the second ones by high construction costs to be financed by government grants or public bodies. 
As a consequence, new neighborhoods are usually poorly accessible by public transport, thus generating unsustainable mobility behavior, strictly oriented to the exclusive use of private transport.

The proposed methodology would like to overcome this temporal discrepancy, trying to restore the correct connection between land use and the public transport system.

To reach this objective, it is fundamental the sharing of experts of land use (urban developers, architects) and experts on transport systems (transport engineers) in order to match the expected land use of the different areas with the potential offered by the existing transport system or by its short term development.

In particular, we can think to adopt the residual capacity of the mass transit system (existing or its short term development) as a variable that can indicate the location and the magnitude of new residential and activities developments, so that the resulting trips are just directed to use this remaining capacity.

It means that, in such developments, users may be more likely to use public transport and thus more oriented to a sustainable mobility.

This method can be articulated into two main steps:

1. the estimation of the transit demand (its magnitude and location in terms of generated and attracted trips) based on the residual capacity of the mass transit system;

2. the conversion of the transit demand from the generated and attracted trips of step 1 to the quantity and location of residential and business volumes.

The first step can be seen as an Origin-Destination Matrix Estimation problem (ODME, Cascetta [6], Cipriani et al. [7]), consistent with the capacities of the links of the mass transit system network. Alba et al. [8] formalized it in the case of traffic of private vehicles as:

$\min \sum_{i=1}^{A} w^{a}\left(V^{a}-s \sum_{i=1}^{N} \sum_{j=1}^{N} p_{i j} x_{i} y_{i} T_{i j}^{*}\right)^{2}+z\left(\sum_{i=1}^{N} \sum_{j=1}^{N}\left(T_{i j}^{*}-x_{i} y_{i} T_{i j}^{*}\right)\right)^{2}$

where:

$V^{a}=$ maximum desired flow on link $a$

$T_{i j}{ }^{*}=$ demand value from origin $i$ to destination $j$

$p_{i j}=$ fraction of the demand from $i$ to $j$ using link $a$

$w^{a}, z=$ external weights

$s=$ scale factor

$x_{i}, y_{i}=$ maximum level of variation for origin $i$ and destination $j$

$N=$ number of zones.

The first term indicates the difference between the expected link value and its actual value, while the second term adds the difference between the starting demand matrix and the final one. So it is looking for a demand matrix not too far from its starting configuration and leading to link values as closest as possible to the expected values. 
In our case, the maximum desired flow on link $a, V^{a}$, is the capacity of the mass transit system, while the output of the procedure is the transit demand matrix $T_{i j}{ }^{*} x_{i} y_{j}$. Once obtained $T_{i j}{ }^{*} x_{i} y_{j}$, the changes in emission and attraction trips can be evaluated respectively by the $x_{i}$ and $y_{j}$ variables.

Obtaining these two variables $\left(x_{i}, y_{j}\right)$ is essential to pass to the step 2 of the method: in fact they are the main input in order to trace back the demand models (emission and attraction models) and to determine possible changes in the development of residences and activities for each existing zone of the study area.

The optimization problem reported in (1) can be solved using purely the capacity of the mass transit system.

However not just the presence (mass transit stop in the area) and availability (available capacity) of the transit service could ensure its use; to encourage the modal shift to the public transport, it is necessary that the transit service is well connected to the rest of the transport network, and most of all that it is accessible.

To take into account the accessibility of the mass transit system, it could be possible to adopt in the proposed method an "effective capacity".

The "effective capacity" is a reduced capacity for each link, computed considering the real accessibility to the different stops of the mass transit system:

$$
V_{e f f}^{a}=\alpha V^{a}
$$

with the $\alpha$ coefficient variable into the interval $(0,1]$. The zero value is not included, because it means an effective capacity equal to zero and it could not be admitted for the demand estimation procedure in (1).

The accessibility to each stop of the mass transit system can be computed considering one or all the following measures:

1. the walking accessibility to the stop: it measures how the road network in the surrounding area of the stop is pedestrian oriented (Pedestrian Oriented Development, POD (Gori et al. [9]);

2. the transit accessibility to the stop: it measures the coverage and the efficacy of the secondary transit services (bus lines) converging to the stops of the mass transit system;

3. the car accessibility to the stop: it measures the possibility to reach the stop of the mass transit system and to park the car in order to continue the travel using the mass transit system (Park and Ride availability).

In this paper, the walking accessibility has been firstly considered in order to compute the effective Capacity: for each stop, a circular area of 800 meters radius has been defined (as the maximum walking area reported by Gori et al. [9] and walkability indicators can be computed considering the road network in each area.

The walkability indicators can be after compared with the benchmark values reported in literature (Gori et al. [9]) in order to trace the reduction of accessibility (the $\alpha$ coefficient) as a function of the walkability indicator. 
Values of walkability indicators equal or greater than the benchmark correspond to an $\alpha$ value of 1 . For walkability indicators lower than the benchmark an exponential reduction of the $\alpha$ coefficient can be assumed (Fig.1).

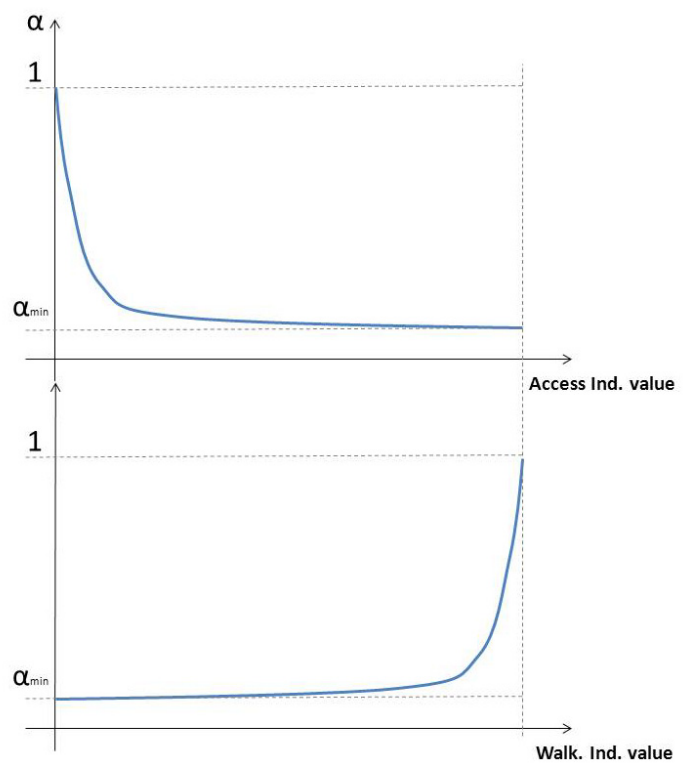

Figure 1: Trend of the alpha coefficient as a function of accessibility indicators.

\section{The application to the city of Rome}

The methodology previously reported has been applied in the present paper to the case of the mass transit network in Rome (Italy).

The urban area of Rome is actually characterized by a population of 3 million with 1.1 million employees, contributing to about 552,000 trips in the morning peak hour. A first division of the city can be done considering areas to be inside or outside the GRA (a circular freeway of approximately $68 \mathrm{~km}$ of length). In regard to the transit system, there are three metro lines (A, B, B1) extending for a total of $45 \mathrm{~km}$. These lines have a radial structure with the main interchange in the city centre (Termini rail station). Other seven rail lines connect the surrounding urban areas to the city centre, but these services are actually far from be frequent and only three of them present an headway lower or equal than 15 minutes in the morning peak hour. The union of five of these rail lines creates a half circle inside the GRA known as the "rail ring".

The analyzed scenario to apply the methodology is the foreseen transit infrastructural scenario at 2020: it includes 4 metro lines (A, B, B1, C) for a total length of $70 \mathrm{~km}$ and an efficient urban rail network with a strong increase of the 
frequencies respect to the current rail lines state (from headway of 15 minutes to 5 minutes on average).

\subsection{Experimental design}

Considering the 2020 scenario, in order to apply the method reported in Chapter 2, firstly two transit demand estimation (Est) have been done:

1. Est1: the residual capacity adopted, as the value of the "measured" transit link flow, is the residual capacity of the metro lines;

2. Est2: the residual capacity adopted, as the value of the "measured" transit link flow, is the residual capacity of the metro lines and of the urban rail network.

So, in the first case (Est1), only a subset of the mass transit network is considered, while in the second case (Est2) the structure of the mass transit network is completed so creating a higher "network effect".

After, the density value of nodes has been fixed as a walkability indicator, with the value of 5 nodes/ha as the benchmark for a road network "pedestrian oriented". Considering for each station of the transit lines a circular basin of 800 meters radius (maximum walking distance for the pedestrian access), the density of nodes can be computed.

The $\alpha$ coefficient can be reduced until the minimum value considering the difference of the computed density of nodes respect to the benchmark, obtaining a reduced capacity (the already called "effective capacity”).

So, finally, a last transit demand estimation has been done:

3. Est3: the residual capacity adopted, as the value of the "measured" transit link flow, is the "effective capacity" of the metro lines.

This last case allows us to study what suggests the adopted procedure taking into account the pedestrian accessibility to the transit stops and a partial mass transit network structure (the urban rail network is not considered in Est3).

\subsection{Experimental results}

Starting from Est1, i.e. considering only the residual capacity of the metro lines at 2020, the following results can be summarized after the application of the procedure:

1. the highest increases of generated trips are located inside the main ring road (Grande Raccordo Anulare - GRA) and in particular between the ring rail and the GRA (Fig.2);

2. the increases of generated trips can be translated into an increment of residences (by adopting a simple emission model) and, as a result of the point 1., the areas of greatest development are located between the ring rail and the GRA, where the population density is lower than the central areas, so where there would be the possibility of building;

3. in the central area of the city the capacity of the mass transit system is already reached at the current state and, considering that the metro 
network will continue to have a radial development also in 2020, there is no possibility to obtain considerable increases of generated trips/residences in the city center;

4. a high number of zones, located mainly in the south/south-east area outside the GRA, report an increase of generated trips, but they are usually of very low values (Fig.2);

5. vice versa attracted trips develop mainly outside the GRA (Fig.3): this result is explained by the process itself, since the residual capacity of the transport system in the direction of suburbs (outside GRA) is maximum and the procedure tends to bridge exactly this remaining capacity. Hence the increase of attracted trips in the suburbs, which can be translated in activities development (by adopting a simple attraction model).

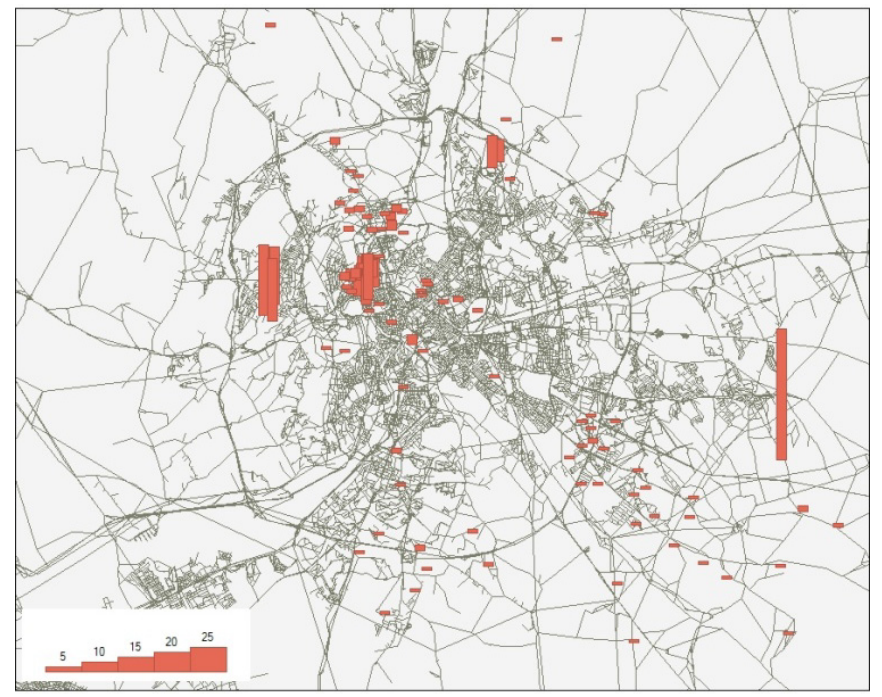

Figure 2: $\quad$ Est1: increase of generated trips.

Passing to Est2, i.e. considering both the residual capacity of the metro lines and of the urban rail network at 2020, the following results can be summarized:

1. also in this case the number of areas increasing their generated trips are higher inside the GRA (Fig.4), but in Est2 they includes also zones located in the city center: this last result underlines the importance of the creation of a network effect between metro and urban rail lines, respect to the only radial structure of the metro lines;

2. also the attracted trips (Fig.5) have the same development obtained in Est1 in order to follow the residual capacity of the mass transit system, i.e. mostly located outside the GRA; 
3. as in Est1, we can translate generated and attracted trips of Est2 into residences and activities, so obtaining location and magnitude where direct the future land-use development.

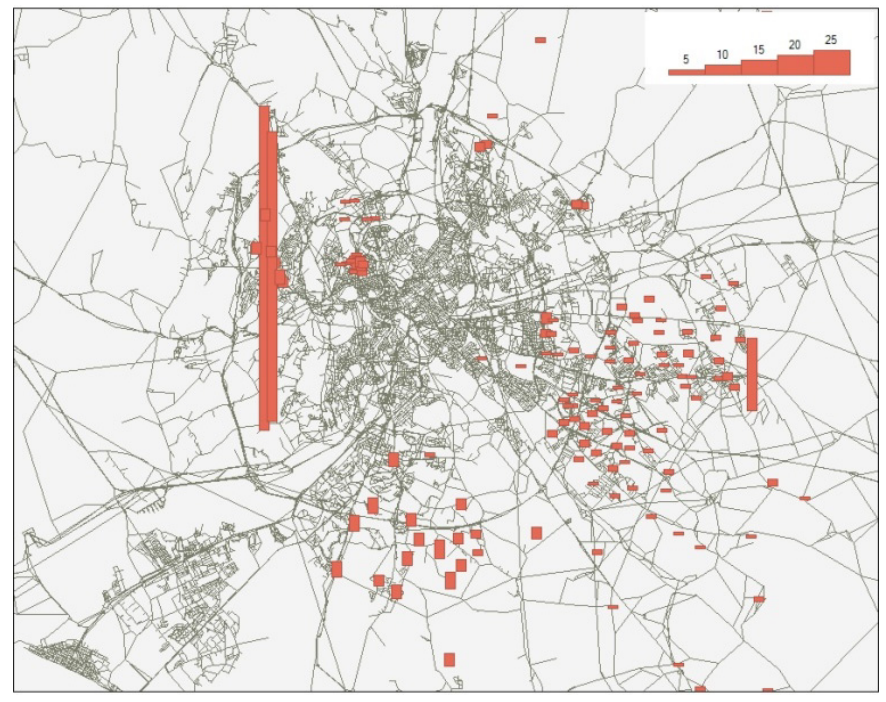

Figure 3: Est1: increase of attracted trips.

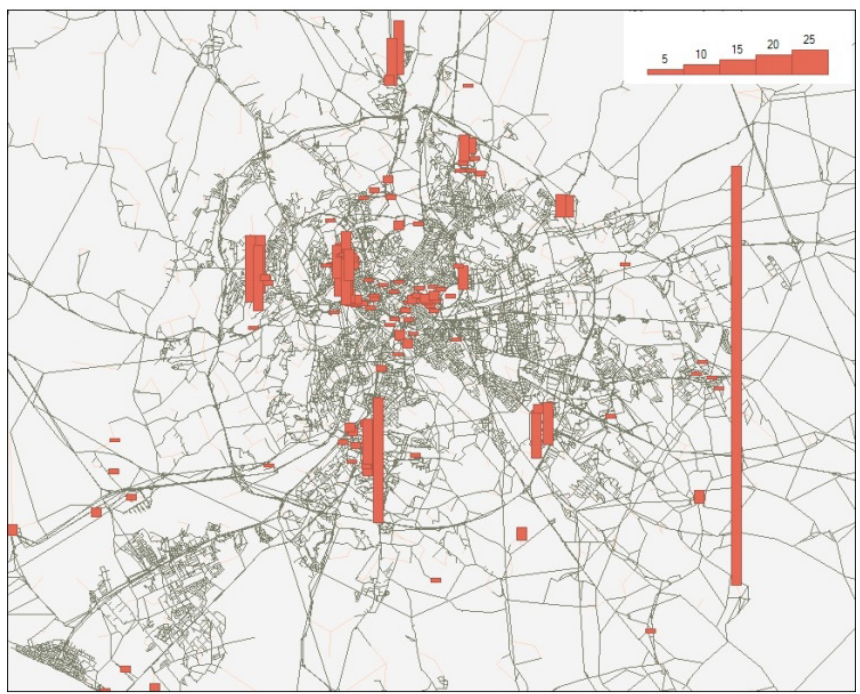

Figure 4: $\quad$ Est 2: increase of generated trips. 


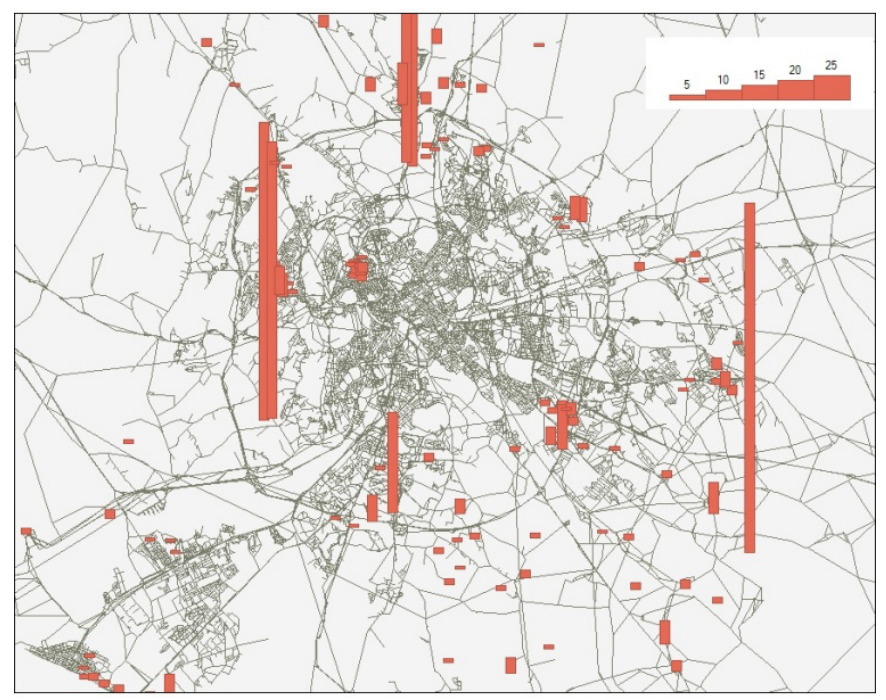

Figure 5: $\quad$ Est 2: increase of attracted trips.

So, Est1 and Est2 report similar results and these results permit to rapidly obtain indications about the possible development of the city in order to exploit all the available capacity of the mass transit system.

This recommended development of the city can be considered sustainable from the point of view of the level of use of the public transport system.

However it has to be sustainable also from the point of view of the single user of the transport system.

If, for example, we consider the result of developing activities in the suburbs of the city, we cannot think that all the trips directed to these suburbs are generated from the central area of the city, according only to an increase of the origin-destination distances (even if well connected by a mass transit network): it is not sustainable for the single user.

Sustainability for the single user means to reduce its distance travelled and its travel times: according to this concept of sustainability, it becomes essential the support of the expert of land use (urban planners, architects) to read the results of the proposed method and evaluate the best way to develop the derived indications.

Coming back to our suburbs, probably the right level of mixed land use has to be searched with the aim that the developed activities can also be used by the inhabitants of the same suburbs.

The "mixed land use" is reported by literature as one of the possible actions in urban areas in order to obtain a sustainable mobility (Cervero and Kochelman, [10]).

After these first set of experiments, the walkability indicator for each station of the metro lines at 2020 has been computed, obtaining an average value for the roman case of 2.02 nodes/ha, with a variation coefficient (ratio between the 
standard deviation and the average value) of 0.29 . From the walkability indicator, the $\alpha$ coefficient has been founded in order to reduce the available capacity of the metro lines as a function of the accessibility. The procedure has been applied to this new scenario (Est3) and the results are reported in Figs 6 and 7.

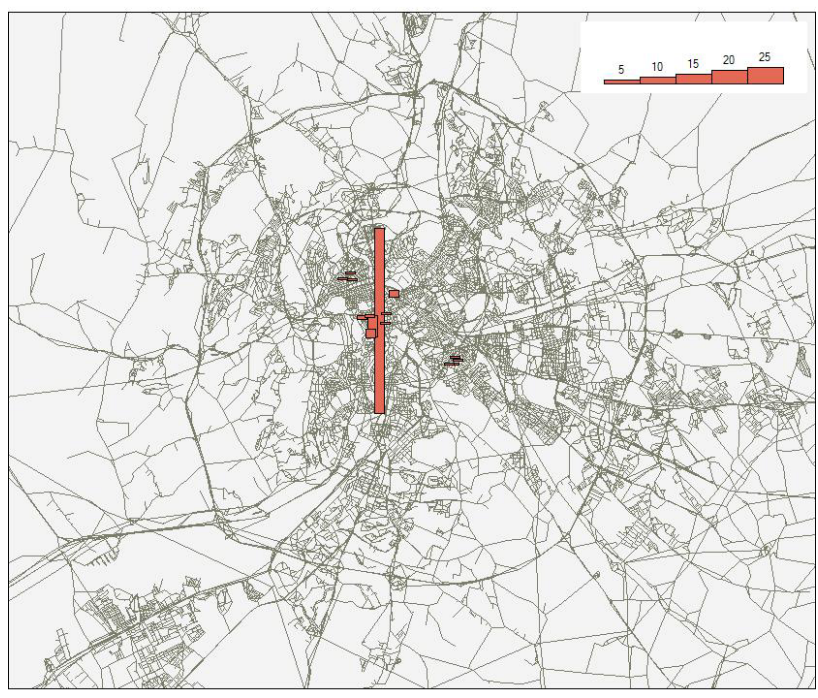

Figure 6: $\quad$ Est3: increase of generated trips.

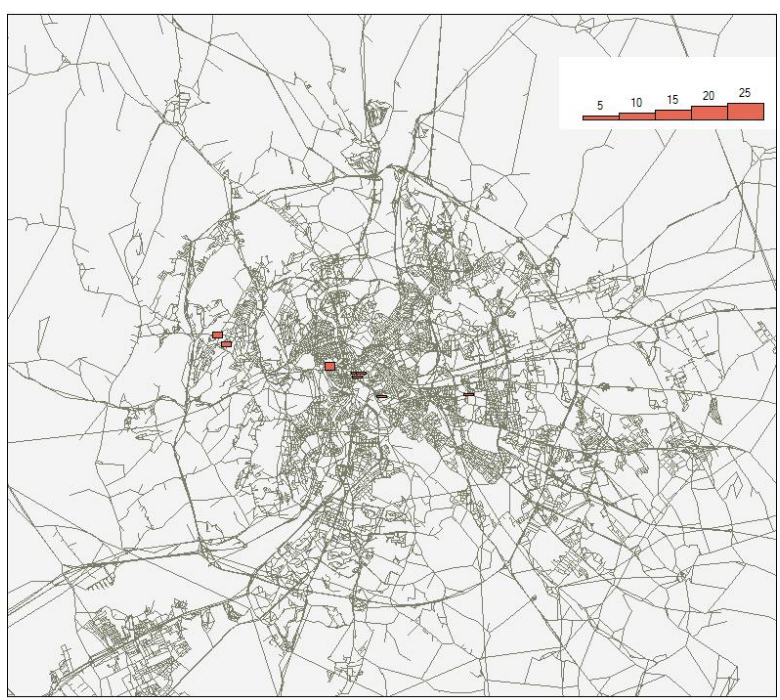

Figure 7: $\quad$ Est3: increase of attracted trips. 
Results show how the current situation of the road network of Rome affects the accessibility to the mass transit stops: the pedestrian network to access to the mass transit stops is in fact largely overlapping with the road network.

But the road network in the city of Rome is designed and developed for the exclusive use of cars; the consequence in the proposed method is that few are the zones where it is possible to operate on the land use in order to exploit the capacity of the transit system. These zones are concentrated mainly in the center, where the road network is well connected.

No zones outside the GRA have been considered in this case by the procedure: it reflects the current trend of the new suburban development, with mainly single use areas thought for the private transport.

\section{Conclusions}

The present study proposes a new method to effectively exploit the transit network, modifying the usual planning approach: the method would like to overcome the temporal discrepancy between infrastructural actions on land use and infrastructural actions on the transport system, using the residual capacity of the mass transit system (existing or its short term development) as a variable to indicate the location and the magnitude of new residential and activities developments.

The method has been articulated into two main steps: the first one is the estimation of the transit demand (its magnitude and location in terms of generated and attracted trips) based on the residual capacity of the mass transit system, while the second one is the conversion from the generated and attracted trips to the quantity and location of residential and business volumes.

The method has been applied to the city of Rome (Italy) and interesting results have been founded from this application: a part from the essential indications about where and how to build, it shows that the development of a network effect for the mass transit system and the accessibility to the transit stops are fundamental variables, not only for the use of the transit system, but also for the correct development of the city.

The proposed procedure demonstrates its capability to be a reliable decision support system, suggesting how the Local Authority could guide the development of the urban area in a sustainable way for the next years.

Future developments of the work need the sharing of experts of land use (urban developers, architects) and experts of transport systems (transport engineers) in order to bind and properly weigh the basic results of the proposed procedure.

\section{References}

[1] Vuchic, V.R. (1999) Transportation for Livable Cities - Rutgers Center for Urban Policy Research.

[2] Banister, D. (2005) Unsustainable Transport: City Transport in the New Century, Routledge, London. 
[3] Bernick, M., Cervero, R. (1997) Transit Villages in the 21st Century, McGraw-Hill, New York, 1997.

[4] Cervero, R. (1998) The Transit Metropolis: A Global Inquiry, Island Press, Washington, D.C.

[5] Gori, S., Nigro, M., Petrelli, M. (2012) The impact of land use characteristics for sustainable mobility: the case study of Rome, European Transport Research Review (2012) 4:153-166.

[6] Cascetta, E. (2009) Transportation systems analysis: models and applications. Springer, New York (NY), USA.

[7] Cipriani, E., Florian, M., Mahut, M., Nigro, M. (2011), “A gradient approximation approach for adjusting temporal origin-destination matrices”. Transportation research. Part c, emerging technologies, vol. 19, p. 270-282, 2011, ISSN: 0968-090X, doi: 10.1016/j.trc.2010.05.013.

[8] Alba, A.C., Beimborn, E., Horowitz, A.J. (2007), "Methods to assess landuse and transportation balance”, 11th TRB National Transportation planning applications conference.

[9] Gori, S., Nigro, M., Petrelli, M. (2012), Indicatori per la valutazione della pedonalità di un'area urbana, in Vivere e camminare in città - mobilità sostenibile e sicurezza stradale, a cura di Michèle Pezzagno e Silvia Docchio, Egaf edizioni, ISBN 978-88-8482-448-6.

[10] Cervero, R., Kochelman, K.M. (1997), “Travel Demand and the three Ds: Density, Diversity and Design”, Transportation Research D, Vol. 2, 199219. 